Journal of Mathematics and Informatics

Vol. 19, 2020, 93-114

ISSN: 2349-0632 (P), 2349-0640 (online)

Published 25 September 2020

www.researchmathsci.org

DOI: http://dx.doi.org/10.22457/jmi.v19a09181

Journal of

Mathematics and

Informatics

\title{
A Deterministic Mathematical Model for the Control of Spread of Prosopis Juliflora Plants
}

\author{
Joel Simon $^{1 *}$, Silas Mirau ${ }^{1}$ and Livingstone S Luboobi ${ }^{2}$ \\ ${ }^{1}$ Department of Applied Mathematics and Computational Science \\ The Nelson Mandela African Institution of Science and Technology \\ P.O.BOX 447, Arusha, Tanzania. Email silas.mirau@ nm-aist.ac \\ ${ }^{2}$ Institute of Mathematical Sciences, Strathmore University \\ 59857-00200, Nairobi, Kenya. Email luboobi@ gmail.com \\ *Corresponding author, E-mail: sjoel698@gmail.com
}

Received 15 August 2020; accepted 21 September 2020

\begin{abstract}
Prosopis juliflora plants are the most aggressive invasive species in the world. They spread by animal movement crossing from one place land to another. In this paper a deterministic model to examine the dynamics of Prosopis julifrola plants is formulated and presented by adopting a similar approach of a dynamical system as used in epidemiological modeling. The local and global stability analyses of the equilibrium points of the model performed by using next-generation for the basic reproduction number $R_{0}$ computation and Lypunov function method. The finding from the study showed that the Prosopis free equilibrium of the model is both locally and globally asymptotically stable if and only if the number of secondary infections, is less than unit, that is $R_{0}<1$. Furthermore, the study showed that there exist Prosopis endemic equilibrium for the spread when $R_{0}>1$. The numerical simulation implemented in MATLAB ODE45 algorithm for solving linear ordinary differential equations. The study findings showed that as the number of ingested animals increase, the plant spread increases on land. Based on the findings, the study recommend the application of the model on endemic areas to improve through: Awareness on animal feeding the plant, provision of insight on plant invasion to policy makers and environmental stakeholders to include in environment framework, seminars and environment clubs by visiting community groups an educating them on plant invasion, through this the plant eradication could be achieved.
\end{abstract}

Keywords: Prosopis juliflora plants, mathematical model, Equilibria, Stability, Numerical simulation

AMS Mathematics Subject Classification (2010): 93A30, 00A71

\section{Introduction}

Prosopis juliflora plants are invasive species in the world originated in Latin America and introduced to Tanzania in 1980's from Taveta Count in Kenya [13]. The plant has invaded Uganda, Kenya and Ethiopia in 1970's. The plant is spread by grazing animals through 


\section{Joel Simon, Silas Mirau and Livingstone S Luboobi}

their movement from one place of land to another as a major agency. However, people, rainfall, wind and floods can spread the plants [1,2,] . In Tanzania, the areas which have been affected by plants are: Mwanza, Arusha, Kilimanjaro, Dodoma, Tanga and Morogoro.With regards to challenges brought by the plant on environment, it displaces native vegetation and absorption of water sources hard accessibility to economic activities such as: Agricultural, fishing, pastoralism and disaster to biodiversity [3, 13]. These challenges brought the need to magnify the efforts to be done upon investigating the magnitude of plant dynamics. However, there are some initiatives that has been taking places to the invaded places on reducing and prevention measure through using chemical and physical means by Center of Agriculture Bioscience International (CABI) to single centers projects in Tanzania, Kenya and Ethiopia. South Africa published National Management Strategies against this invasive plant. Despite of strategies based on statistical and social context, there is no deterministic model that has been employed to this challenge. Most studies $[4,5,6]$ have been done on addressing the problem on life science and the dynamics of invasive species basing on statistics and optimization context while others studies [7-9]. [8], developed a mathematical model for estimating the rate of Prosopis juliflora plants expansion in the Far region in Ethiopia by using Least square method and secondary data for twenty years. Through this fact, this study decided to use a deterministic model for the control the spread of the plant.

The study aims at presenting the new method of deterministic model by using details of variables and parameters, assumptions and model formulation in section 2. In section three concentrate on calculation of basic of Reproduction number, Prosopis free equilibrium, local and global stability analyses and sensitivity. Section four is where results and discussions, numerical analysis of the model is done and finally we conclude with recommendations.

\section{Materials and methods}

The study involved model formulation in the first phase and model analysis in the second phase. Moreover, the assessment of various parameters on the plant spread was conducted. A mathematical model considered the interactions between land and animal populations in the spread of Prosopis julifrola plant. The spread process is denoted by three compartments for the land and two for animal population. In this case, we considered the approach which is similar to dynamical system as applied in epidemiological modeling and ecological modeling. The grazing animals are considered as the main contributor to the spread of the plant to unaffected pieces of land.

\subsection{Model formulation}

In this study, we consider a method similar to that used in the studies of [10-12]. The significance in formulating a mathematical model of a given real life situation in our daily life is very useful in giving great understanding of the situation. For the dynamics of Prosopis juliflora plant. The model is formulated by using the two populations particularly land and animals. The land involves three compartments, which are: susceptible land ( $S_{L}$ ), invaded land ( $\left.I_{L}\right)$ and the reclaimed land $\left(R_{L}\right)$; whereas for the animals were susceptible animals $\left(S_{A}\right)$ and infestation animals $\left(I_{A}\right)$. The susceptible land compartment $\left(S_{L}\right)$ refer to the piece of land which is not yet invaded by the plant. The invaded land, compartment 
A Deterministic Mathematical Model for the Spread of Prosopis Juliflora Plants

is the number of acres of land which has the seeds of plant that have already germinated and need to be restored; Reclaimed land compartment $\left(R_{L}\right)$ are portions of land in acres that has been invaded by the plant but is successfully being recovered by physical removal, application of chemicals, harvesting, application of leaves sprays to the plant and limitations on the plant growing excessively. As regards to animal population there are susceptible animals $\left(S_{A}\right)$ which are cattle grazing in the invaded land and so are prone in ingesting the plant seeds on the land. The infected animal $\left(I_{A}\right)$, are cattle that have already ingested seeds of the plant and they are the ones that will drop dung containing seeds on land.

\subsection{Model assumptions}

In our model formulation, we make the following assumptions:

i. The plant seeds are majorly spread to susceptible land portions by grazing animals;

ii. Animal mixing is homogeneous in the considered ecosystem;

iii. The occurrence of natural calamities can take place at any compartment of land population;

iv. Recruitment of animals into the system is only through susceptible animal compartment by birth;

v. The land restoration takes place only on the invaded land;

vi. The land is claimed from susceptible acres of land.

In Table 1 and Table 2 the variables and parameters used in the model are respectively defined.

Table 1: The five variables for both land and animal populations

\begin{tabular}{|c|c|}
\hline Variable & Description \\
\hline$S_{L}$ & Number of acres of land which is not yet invaded \\
\hline$I_{L}$ & $\begin{array}{l}\text { Number of acres of land that has already germinated and need to be } \\
\text { restored }\end{array}$ \\
\hline$R_{L}$ & $\begin{array}{l}\text { Acres of land that has been invaded by the plant but is successfully } \\
\text { recovered by various measures }\end{array}$ \\
\hline$S_{A}$ & Number of animals not yet infested with plant seed \\
\hline$I_{A}$ & Number of animals that have ingested plant seeds \\
\hline
\end{tabular}

\subsection{Flow diagram chart}

Basing on the dynamics of Prosopis juliflora plant in animal population and land according to the assumptions made, the flow diagram for the interactions between the plant, the land and the animals is as in Figure 2.

$\beta_{2} I_{L} S_{A}$ describes the spread rate of Prosopis juliflora plant after interaction between infested land and susceptible animals $\beta_{1} I_{A} S_{L}$ describes the spread rate of Prosopis juliflora plant after interaction between susceptible land and infected animals. 
Joel Simon, Silas Mirau and Livingstone S Luboobi

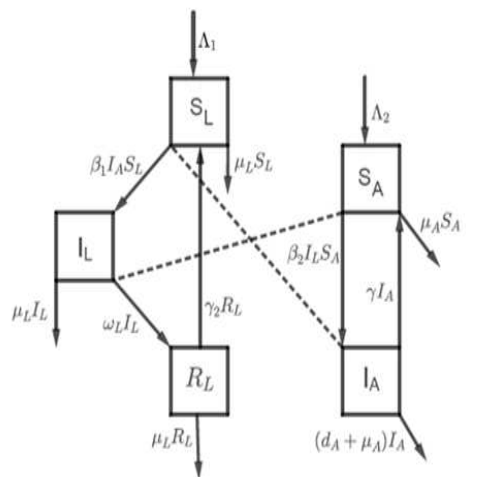

Figure 1: The flow diagram for the spread of Prosopis juliflora plant on piece of land by animals that ingest the plant seeds.

Table 2: Parameters and its Description

\begin{tabular}{ll}
\hline Parameter & Description \\
\hline$\Lambda_{1}$ & Reclamation rate into the susceptible acres of land portions population \\
$\Lambda_{2}$ & Per capita birth rate of animal population \\
$\mu_{A}$ & Per capita natural death rate of animal population \\
$d_{A}$ & Per capita death rate of animal population induced by ingested seed \\
$\mu_{L}$ & $\begin{array}{l}\text { Land portion used for constructions project and occurrence of natural } \\
\text { calamities }\end{array}$ \\
$\omega_{L}$ & Rate of progression from invaded land to the reclaimed land \\
$\gamma$ & $\begin{array}{l}\text { Rate of recovery or restoration of infested animal into susceptible animal } \\
\gamma_{2}\end{array}$ \\
\hline
\end{tabular}

Model equations. Through compartmental consideration, as in Figure 1 depicts, we formulated the basic mathematical model which demonstrates the dynamics for Prosopis juliflora plant using the following differential equations:

$$
\left\{\begin{array}{l}
\frac{d S_{L}}{d t}=\Lambda_{1}+\gamma_{2} R_{L}-\beta_{1} I_{A} S_{L}-\mu_{L} S_{L} \\
\frac{d I_{L}}{d t}=\beta_{1} I_{A} S_{L}-\mu_{L} I_{L}-\omega_{L} I_{L} \\
\frac{d R_{L}}{d t}=\omega_{L} I_{L}-\gamma_{2} R_{L}-\mu_{L} R_{L} \\
\frac{d S_{A}}{d t}=\Lambda_{2}+\gamma I_{A}-\beta_{2} I_{L} S_{A}-\mu_{A} S_{A} \\
\frac{d I_{A}}{d t}=\beta_{2} I_{L} S_{A}-\gamma I_{A}-\left(d_{A}+\mu_{A}\right) I_{A}
\end{array}\right.
$$


A Deterministic Mathematical Model for the Spread of Prosopis Juliflora Plants with initial conditions:

$$
\begin{aligned}
& S_{L}(0)>0, I_{L}(0) \geq 0, R_{L}(0) \geq 0, \\
& S_{A}(0)>0, I_{A}(0) \geq 0
\end{aligned}
$$

The total size of land is given by: ${ }_{N}=S_{L}+I_{L}+R_{L}$ while total number of animal is given by: $N_{A}=S_{A}+I_{A}$

\section{Basic properties of the model}

Under this section, we checked if our model is mathematically and epidemiologically well posed. Furthermore, we are check the existence of boundedness of the solution.

\subsection{Non negativity of solutions}

We consider equations for $S_{L}, I_{L}, R_{L}$ for land;

Working with the reclaimed land: $R_{L}$

Recalling derivative for reclaimed land from System of differential equation (1) as shown below:

$$
\begin{aligned}
& \text { Thus: } \frac{d R_{L}}{d t}=\omega_{L} I_{L}-\gamma_{2} R_{L}-\mu_{L} R_{L} \\
& \frac{d R_{L}}{d t}=\omega_{L} I_{L}-\left(\gamma_{2}+\mu_{L}\right) R_{L} \\
& \text { From: } I_{L}(t) \geq 0 \\
& \frac{d R_{L}}{d t} \geq-\left(\gamma_{2}+\mu_{L}\right) R_{L} \\
& \frac{d R_{L}}{R_{L}} \geq-\left(\gamma_{2}+\mu_{L}\right) d t \\
& \int_{R_{L(0)}}^{R_{L(t)}} \frac{d R_{L}}{R_{L}} \geq-\int_{0}^{t}\left(\gamma_{2}+\mu_{L}\right) d t \\
& \ln \left(\frac{R_{L}(t)}{R_{0}(0)}\right) \geq-\left(\gamma_{2}+\mu_{L}\right) t \\
& \left(\frac{R_{L}(t)}{R_{0}(0)}\right) \geq e^{-\left(\gamma_{2}+\mu_{L}\right) t} \\
& R_{L}(t) \geq R_{0}(0) e^{-\left(\gamma_{2}+\mu_{L}\right) t} \geq 0
\end{aligned}
$$

Thus, $R_{L} \geq 0$

For Susceptible land;

$$
\frac{d S_{L}}{d t}=\Lambda_{1}+\gamma_{2} R_{L}-\beta_{1} I_{L} S_{L}-\mu_{L} S_{L}
$$


Joel Simon, Silas Mirau and Livingstone S Luboobi

$$
\begin{gathered}
\frac{d S_{L}}{d t}>-\beta_{1} I_{A} S_{L}-\mu_{L} S_{L} \\
\frac{d S_{L}}{d t}>-\left(\beta_{1} I_{A}+\mu_{L}\right) S_{L} \\
\frac{d S_{L}}{S_{L}}>-\left(\beta_{1} I_{A}+\mu_{L}\right) d t \\
\int_{S_{L}(0)}^{S_{L}(t)} \frac{d S_{L}}{S_{L}}>-\int\left(\beta_{1} I_{A}+\mu_{L}\right) d t \\
\ln \left(\frac{S_{L}(t)}{S_{L}(0)}\right)>-\left(\beta_{1} I_{A}+\mu_{L}\right) t \\
\frac{S_{L}(t)}{S_{L}(0)}>e^{-\left(\beta_{1} I_{A}+\mu_{L}\right) t} \\
S_{L}(t)>S_{L}(0) e^{-\left(\beta_{1} I_{A}+\mu_{L}\right) t}>0 \\
S_{L}(t)>0
\end{gathered}
$$

Through applying the same technique, we get:

$$
S_{L}(t), I_{L}(t), R_{L}(t), S_{A}(t), I_{A}(t) \geq 0
$$

\subsection{Invariant region}

Invariant region is the one which shows the boundedness of the solution. To determine the region, the animal and land population we considered separately.

For land:

$$
N_{L}=S_{L}+I_{L}+R_{L}
$$

Differentiating size of land compartment, we get:

$$
\begin{aligned}
& \quad \frac{d N_{L}}{d t}=\frac{d\left(S_{L}+I_{L}+R_{L}\right)}{d t}=\Lambda_{1}+\gamma_{2} R_{L}-\beta_{1} I_{L} S_{L}-\mu_{L} S_{L} \\
& \frac{d N_{L}}{d t}=\frac{d\left(S_{L}+I_{L}+R_{L}\right)}{d t}=\Lambda_{1}+\gamma_{2} R_{L}-\beta_{1} I_{L} S_{L}-\mu_{L} S_{L} \\
& \frac{d N_{L}}{d t}=\frac{d S_{L}}{d t}+\frac{d I_{L}}{d t}+\frac{d R_{L}}{d t}=\Lambda_{1}-\mu_{L} S_{L}-\mu_{L} I_{L}-\mu_{L} R_{L} \\
& \frac{d\left(S_{L}+I_{L}+R_{L}\right)}{d t}=\Lambda_{1}-\left(S_{L}+I_{L}+R_{L}\right) \mu_{L} \\
& \frac{d N_{L}(t)}{d t}=\Lambda_{1}-N_{L}(t) \mu_{L}
\end{aligned}
$$


A Deterministic Mathematical Model for the Spread of Prosopis Juliflora Plants

$$
\frac{d N_{L}(t)}{d t}+\mu_{L} N_{L}(t)=\Lambda_{1}
$$

Equation (3) is the linear ode. Now, to obtain the solution, integrating factor is applied.

Thus the integrating factor $I=e^{\int \mu_{L} d t}=e^{\mu_{L} t}$

Multiplying equation (3) with integrating factor we get;

$$
\begin{gathered}
e^{\mu_{L} t} \frac{d N_{L}(t)}{d t}+\mu_{L} N_{L}(t) e^{\mu_{L} t}=\Lambda_{1} e^{\mu_{L} t} \\
\frac{d}{d t}\left(\mu_{L} N_{L}(t) e^{\mu_{L} t}\right)=\Lambda_{1} e^{\mu_{L} t} \\
d\left(\mu_{L} N_{L}(t) e^{\mu_{L} t}\right)=\Lambda_{1} e^{\mu_{L} t} d t
\end{gathered}
$$

Integrating equation (4) in both side we get:

$$
\begin{array}{r}
\int d\left(\mu_{L} N_{L}(t) e^{\mu_{L} t}\right)=\int \Lambda_{1} e^{\mu_{L} t} d t \\
N_{L}(t)=\frac{\Lambda_{1}}{\mu_{L}}+C e^{-\mu_{t} t}
\end{array}
$$

By computing $C$ at $t=0$, we get;

$$
C=N_{L}(0)-\frac{\Lambda_{1}}{\mu_{L}}
$$

By substituting $C$ equation 5 we get:

$$
N_{L}(t)=\frac{\Lambda_{1}}{\mu_{L}}+\left(N_{L}(0)-\frac{\Lambda_{1}}{\mu_{L}}\right) e^{-\mu_{t} t}
$$

Now through considering two cases;

$$
N_{L}(0)>\frac{\Lambda_{1}}{\mu_{L}} \text {, or } N_{L}(0)<\frac{\Lambda_{2}}{\mu_{L}} \text {. }
$$

Now, the boundedness condition is $N_{L}(t) \leq \max \left\{N_{L}(0), \frac{\Lambda_{1}}{\mu_{L}}\right\}$

Applying the same technique to the remaining equation, we get:

Now through considering two cases:

$$
N_{A}(t) \leq \frac{\Lambda_{2}}{\mu_{A}}+\left(N_{A}(0)-\frac{\Lambda_{2}}{\mu_{A}}\right) e^{-\mu_{A} t}
$$

Thus:

For animal population, the boundedness condition is:

$$
N_{A}(0)>\frac{\Lambda_{2}}{\mu_{A}}, \text { or } N_{A}(0)<\frac{\Lambda_{2}}{\mu_{A}} .
$$


Joel Simon, Silas Mirau and Livingstone S Luboobi

$$
N_{A}(t) \leq \max \left\{N_{A}(0), \frac{\Lambda_{2}}{N_{A}}\right\}
$$

Therefore the model system (1) is positive invariant in the region:

$$
\Omega=\left\{S_{L}, I_{L}, R_{L}, S_{A}, I_{A}\right\} \in R^{5}: N_{L}(t) \leq \max \left\{N_{L}(0), \frac{\Lambda_{1}}{\mu_{L}}\right\}, N_{A}(t) \leq \max \left\{N_{A}(0), \frac{\Lambda_{2}}{N_{A}}\right\}
$$

\subsection{Model analysis}

In this analysis we to find the existence of Prosopis free equilibrium for the Prosopis dynamics and computation of its Reproduction number.

\subsubsection{Existence of prosopis free equilibrium (PFE) point}

Now PFE is obtained when we set derivatives equal to zero and $I_{L}=I_{A}=R_{L}=0$ and hence we solve for $S_{L}$ and $S_{A}$ as follows:

$$
\begin{aligned}
& \frac{d S_{L}}{d t}=\frac{d S_{A}}{d t}=\frac{d R_{L}}{d t}=\frac{d I_{A}}{d t}=\frac{d I_{L}}{d t}=0 \\
& \left\{\begin{array}{l}
\Lambda_{1}-\beta_{1} S_{L} I_{A}-\mu_{L} S_{L}+\gamma_{2} R_{L}=0 \\
\beta_{1} S_{L} I_{A}-\left(\mu_{L}+\omega_{L}\right) I_{L}=0 \\
\omega_{L} I_{L}-\left(\gamma_{2}+\mu_{L}\right) R_{L}=0 \\
\Lambda_{2}-\beta_{2} S_{A} I_{L}+\gamma I_{L}-\mu_{A} S_{A}=0 \\
\beta_{2} S_{A} I_{L}-\gamma I_{A}-\left(d_{A}+\mu_{A}\right) I_{A}=0 \\
\Lambda_{1}-\mu_{L} S_{L}=0, \text { that is: } S_{L}=\frac{\Lambda_{1}}{\mu_{L}}
\end{array} \quad \text { and } \quad \Lambda_{2}-\mu_{A} S_{A}=0 \text {, that is: } S_{A}=\frac{\Lambda_{2}}{\mu_{A}}\right.
\end{aligned}
$$

Therefore, there exist a Prosopis free equilibrium (PFE) $E^{0}\left(S_{L}^{0}, I_{L}^{0}, R_{L}^{0}, S_{L}^{0}, I_{L}^{0}\right)$ which is equal to $\left(\frac{\Lambda_{1}}{\mu_{L}}, 0,0, \frac{\Lambda_{2}}{\mu_{A}}, 0\right)$

\subsubsection{The prosopis endemic equilibrium point (PEE)}

The Prosopis endemic equilibrium PEE of the model system (1) $E^{*}$ is steady solution in which the spread of the plant persists and in the solution $I_{L} \neq 0, R_{L} \neq 0, I_{A} \neq 0$.

Now from equation system (1) all derivatives are set equal to zero.

Let $E^{*}=\left(S_{L}{ }^{*}, I_{L}{ }^{*}, R_{L}{ }^{*}, S^{*}{ }_{A}, I_{A}{ }^{*}\right)$ be the equilibrium and performing computations as follows: 
A Deterministic Mathematical Model for the Spread of Prosopis Juliflora Plants

From system (1) for ingested animal, we get:

$\beta_{2} S_{A} I_{L}-\left(\gamma+d_{A}+\mu_{A}\right) I_{A}=0$

Thus:

$I_{A}{ }^{*}=\frac{\beta_{2} \Lambda_{1} \Lambda_{2}}{\gamma\left(\mu_{L}+\omega_{L}\right)\left(\gamma_{2}+\mu_{L}+\omega_{L}\right)+\left(d_{A}+\mu_{A}\right)\left(\beta_{2} \Lambda_{1}+\mu_{A} \mu_{L}\left(\mu_{L}+\omega_{L}\right)\left(\gamma_{2}+\mu_{L}+\omega_{L}\right)\right)}$

From system (1) for infested land, we get:

$$
\beta_{1} S_{L} I_{A}-\left(\mu_{L}+\omega_{L}\right) I_{L}=0 \geq I_{L}^{*}=\frac{\Lambda_{1}}{\mu_{L}\left(\left(\mu_{L}+\omega_{L}\right)\left(\gamma_{2}+\mu_{L}+\omega_{L}\right)\right)}
$$

From system (1) for claimed land, we get: $\omega_{L} I_{L}-\left(\gamma_{2}+\mu_{L}\right) R_{L}=0 \geq R_{L}^{*}=\frac{\omega_{L} I_{L}^{*}}{\gamma_{2}+\mu_{L}}$

From system (1) for susceptible land, we get:

$$
\Lambda_{1}-\beta_{1} S_{L}{ }^{*} I_{A}{ }^{*}-\mu_{L} S_{L}{ }^{*}+\gamma_{2}\left(\frac{\omega_{L} I_{L}^{*}}{\gamma_{2}+\mu_{L}}\right)=0 \geq S_{L}^{*}=\frac{\Lambda_{1}\left(\gamma_{2}+\mu_{L}\right)+\gamma_{2} \omega_{L} I_{L}^{*}}{\left(\beta_{1} I_{A}^{*}-\mu_{L}\right)\left(\gamma_{2}+\mu_{L}\right)}
$$

From system (1) for susceptible animal, we get:

$\Lambda_{2}+\gamma I_{A}{ }^{*}-\beta_{2} S_{A}{ }^{*} I_{L}{ }^{*}-\mu_{A} S_{A}^{*}=0$

Thus:

$S_{A}^{*}=\frac{\Lambda_{2}+\gamma I_{A}^{*}}{\beta_{2} I_{L}^{*}+\mu_{A}}$

The values of $\left(S_{L}{ }^{*}, I_{L}{ }^{*}, R_{L}{ }^{*}, S^{*}{ }_{A}, I_{A}{ }^{*}\right)$, as shown above, are positive. Thus there is an exist of Prosopis endemic equilibrium point.

\subsection{Reproduction number of prosopis dynamics}

The basic reproduction number is the average of secondary spread of seed plant by single seed plant when introduced in an entirely susceptible land population. We used approach in [14-16] .It determine whether the spread persists or clear out. The spread clears out when $R_{0}<1$ and persist when

$R_{0}>1$. In computing basic reproduction number, we adopt the method similar to that in epidemiological studies. In this case the next generation matrix method in which new spread and transfer functions are considered. If the new spread is mathematically defined by $f_{i}$ and transfer terms by $v_{i}$, then the matrices $\mathbf{F}$ and $\mathbf{V}$ are given by $\mathbf{F}=\frac{\partial f_{i}}{\partial I_{i}}(X)$ and $\mathbf{V}=\frac{\partial v_{i}}{\partial I_{j}}$ as defined by [17].

By consideration of infested subpopulation which are: 
Joel Simon, Silas Mirau and Livingstone S Luboobi

$$
\left\{\begin{array}{l}
\frac{d I_{L}}{d t}=\beta_{1} S_{L} I_{A}-\left(\mu_{L}+\omega_{L}\right) I_{L} \\
\frac{d I_{A}}{d t}=\beta_{2} S_{A} I_{L}-\left(\gamma+d_{A}+\mu_{A}\right) I_{A}
\end{array}\right.
$$

Now we find matrix $\mathbf{F}$ and matrix $\mathbf{V}$ where by $\mathbf{F}$ contains force of infestation and $\mathbf{V}$, is the remaining terms.

Let $X=\left(I_{L}, I_{A}\right), f_{1}=\beta_{1} S_{L} I_{A}, f_{2}=\beta_{2} S_{A} I_{L}, V_{1}=\left(\mu_{L}+\omega_{L}\right) I_{L}, V_{2}=\left(\gamma+d_{A}+\mu_{A}\right) I_{A}$

$$
\begin{gathered}
f(X)=\left(\begin{array}{l}
f_{1} \\
f_{2}
\end{array}\right) \\
f(X)=\left(\begin{array}{c}
\beta_{1} S_{L} I_{A} \\
\beta_{2} S_{A} I_{L}
\end{array}\right) \text { and } \mathbf{V}(X)=\left(\begin{array}{c}
\left(\mu_{L}+\omega_{L}\right) I_{L} \\
\left(\gamma+d_{A}+\mu_{A}\right) I_{A}
\end{array}\right)
\end{gathered}
$$

By computing partial derivatives on system equation (11) for $\mathbf{F}$ and $\mathbf{V}$ we get:

$$
\begin{gathered}
\mathbf{F}=\left(\begin{array}{ll}
\frac{\partial f_{1}}{\partial I_{L}} & \frac{\partial f_{1}}{\partial I_{A}} \\
\frac{\partial f_{2}}{\partial I_{L}} & \frac{\partial f_{2}}{\partial I_{A}}
\end{array}\right)=\left(\begin{array}{cc}
0 & \beta_{1} S_{L} \\
\beta_{2} S_{A} & 0
\end{array}\right) \\
\mathbf{V}=\left(\begin{array}{ll}
\frac{\partial V_{1}}{\partial I_{L}} & \frac{\partial V_{1}}{\partial I_{A}} \\
\frac{\partial V_{2}}{\partial I_{L}} & \frac{\partial V_{2}}{\partial I_{A}}
\end{array}\right)=\left(\begin{array}{cc}
\mu_{L}+\omega_{L} & 0 \\
0 & \gamma+d_{A}+\mu_{A}
\end{array}\right)
\end{gathered}
$$

Through evaluation of $\mathbf{F}$ and $\mathbf{V}$ at PFE we get:

$$
\begin{gathered}
\mathbf{F}=\left(\begin{array}{cc}
0 & \beta_{1} S^{0}{ }_{L} \\
\beta_{2} S_{A}^{0} & 0
\end{array}\right)=\left(\begin{array}{cc}
0 & \frac{\beta_{1} \Lambda_{1}}{\mu_{L}} \\
\frac{\beta_{2} \Lambda_{1}}{\mu_{A}} & 0
\end{array}\right) \\
\left.\quad \mathbf{V}\right|_{P F E}=\left(\begin{array}{cc}
\mu_{L}+\omega_{L} & 0 \\
0 & \gamma+d_{A}+\mu_{A}
\end{array}\right)
\end{gathered}
$$

Finding the inverse of $\mathbf{V}$ we get: 
A Deterministic Mathematical Model for the Spread of Prosopis Juliflora Plants

$$
\mathbf{V}^{-1}=\frac{1}{\left(\mu_{L}+\omega_{L}\right)\left(\gamma+d_{A}+\mu_{A}\right)}\left(\begin{array}{cc}
\gamma+d_{A}+\mu_{A} & 0 \\
0 & \mu_{L}+\omega_{L}
\end{array}\right)
$$

Then we compute $\mathbf{F V}^{-1}$ we get:

$$
\begin{aligned}
& \mathbf{F V}^{-1}=\left(\begin{array}{cc}
0 & \frac{\beta_{1} \Lambda_{1}}{\mu_{L}} \\
\frac{\beta_{2} \Lambda_{1}}{\mu_{A}} & 0
\end{array}\right)\left(\begin{array}{cc}
\frac{\gamma+d_{A}+\mu_{A}}{\left(\mu_{L}+\omega_{L}\right)\left(\gamma+d_{A}+\mu_{A}\right)} & 0 \\
0 & \frac{\mu_{L}+\omega_{L}}{\left(\mu_{L}+\omega_{L}\right)\left(\gamma+d_{A}+\mu_{A}\right)}
\end{array}\right) \\
& \mathbf{F V}^{-1}=\left(\begin{array}{cc}
0 & \frac{\beta_{1} \Lambda_{1}}{\mu_{L}\left(\gamma+d_{A}+\mu_{A}\right)} \\
\frac{\beta_{2} \Lambda_{2}}{\mu_{A}\left(\mu_{L}+\omega_{L}\right)} & 0
\end{array}\right)
\end{aligned}
$$

We compute the eigenvalues using: $\left|\mathbf{F V}^{-1}-\lambda\right|=0$

$$
\begin{gathered}
=\left(\begin{array}{cc}
-\lambda & \frac{\beta_{1} \Lambda_{1}}{\mu_{L}\left(\gamma+d_{A}+\mu_{A}\right)} \\
\frac{\beta_{2} \Lambda_{2}}{\mu_{A}\left(\mu_{L}+\omega_{L}\right)} & -\lambda
\end{array}\right) \\
\lambda^{2}=\frac{\beta_{1} \beta_{2} \Lambda_{1} \Lambda_{2}}{\mu_{A} \mu_{L}\left(\mu_{L}+\omega_{L}\right)\left(\gamma+d_{A}+\mu_{A}\right)} \\
\lambda= \pm \sqrt{\frac{\beta_{1} \beta_{2} \Lambda_{1} \Lambda_{2}}{\mu_{A} \mu_{L}\left(\mu_{L}+\omega_{L}\right)\left(\gamma+d_{A}+\mu_{A}\right)}}
\end{gathered}
$$

In this case the reproduction number is given by spectral radius $\rho\left(\mathbf{F V}^{-1}\right)$ :

Which gives:

$$
R_{0}=\rho\left(\mathbf{F V}^{-1}\right)=\sqrt{\frac{\beta_{1} \beta_{2} \Lambda_{1} \Lambda_{2}}{\mu_{A} \mu_{L}\left(\mu_{L}+\omega_{L}\right)\left(\gamma+d_{A}+\mu_{A}\right)}}
$$

\subsection{Local stability of Prosopis free equilibrium point (PFE)}

In order to get local stability of (PFE), we have to show that the variation matrix $J\left(E_{0}\right)$ of our model system (1) has negative eigenvalues by computing the differentiation of the system The Prosopis endemic equilibrium PEE of the model system (1) $E^{*}$ is steady solution in which the spread of the plant persists and in the solution $I_{L} \neq 0, R_{L} \neq 0, I_{A} \neq 0$.

Now from equation system $(1)$ all derivatives are set equal to zero.

Let $E^{*}=\left(S_{L}{ }^{*}, I_{L}{ }^{*}, R_{L}{ }^{*}, S^{*}{ }_{A}, I_{A}{ }^{*}\right)$ be the equilibrium and performing computations as follows: 
Joel Simon, Silas Mirau and Livingstone S Luboobi

From system (1) for ingested animal, we get:

$\beta_{2} S_{A} I_{L}-\left(\gamma+d_{A}+\mu_{A}\right) I_{A}=0$

Thus :

$I_{A}{ }^{*}=\frac{\beta_{2} \Lambda_{1} \Lambda_{2}}{\gamma\left(\mu_{L}+\omega_{L}\right)\left(\gamma_{2}+\mu_{L}+\omega_{L}\right)+\left(d_{A}+\mu_{A}\right)\left(\beta_{2} \Lambda_{1}+\mu_{A} \mu_{L}\left(\mu_{L}+\omega_{L}\right)\left(\gamma_{2}+\mu_{L}+\omega_{L}\right)\right)}$

From system (1) for infested land, we get:

$\beta_{1} S_{L} I_{A}-\left(\mu_{L}+\omega_{L}\right) I_{L}=0 \geq I_{L}^{*}=\frac{\Lambda_{1}}{\mu_{L}\left(\left(\mu_{L}+\omega_{L}\right)\left(\gamma_{2}+\mu_{L}+\omega_{L}\right)\right)}$

From system (1) for claimed land, we get: $\omega_{L} I_{L}-\left(\gamma_{2}+\mu_{L}\right) R_{L}=0 \geq R_{L}^{*}=\frac{\omega_{L} I_{L}^{*}}{\gamma_{2}+\mu_{L}}$

From system (1) for susceptible land, we get:

$\Lambda_{1}-\beta_{1} S_{L}{ }^{*} I_{A}{ }^{*}-\mu_{L} S_{L}{ }^{*}+\gamma_{2}\left(\frac{\omega_{L} I_{L}^{*}}{\gamma_{2}+\mu_{L}}\right)=0 \geq S_{L}^{*}=\frac{\Lambda_{1}\left(\gamma_{2}+\mu_{L}\right)+\gamma_{2} \omega_{L} I_{L}^{*}}{\left(\beta_{1} I_{A}^{*}-\mu_{L}\right)\left(\gamma_{2}+\mu_{L}\right)}$

From system (1) for susceptible animal, we get:

$\Lambda_{2}+\gamma I_{A}{ }^{*}-\beta_{2} S_{A}{ }^{*} I_{L}{ }^{*}-\mu_{A} S_{A}{ }^{*}=0$

Thus:

$S_{A}^{*}=\frac{\Lambda_{2}+\gamma I_{A}^{*}}{\beta_{2} I_{L}^{*}+\mu_{A}}$

The values of $\left(S_{L}{ }^{*}, I_{L}{ }^{*}, R_{L}{ }^{*}, S^{*}{ }_{A}, I_{A}{ }^{*}\right)$, as shown above, are positive. Thus there is an exist of Prosopis endemic equilibrium point with respect to $\left(S_{L}, I_{L}, R_{L}, S_{A}, I_{A}\right)$ at the Prosopis free equilibrium which gives:

$J\left(E_{0}\right)=$

$\left(\begin{array}{ccccc}-\left(\beta_{1} I_{L}+\mu_{L}\right) & -\beta_{1} S_{L} & \gamma_{2} & 0 & 0 \\ \beta_{1} S_{L} & -\left(\mu_{L}+\omega_{L}\right) & 0 & 0 & 0 \\ 0 & \omega_{L} & -\left(\gamma_{2}+\mu_{L}\right) & 0 & 0 \\ 0 & -\left(\beta_{2} S_{A}\right) & 0 & -\left(\beta_{2} I_{L}+\mu_{L}\right) & \gamma \\ 0 & \beta_{1} S_{A} & 0 & \beta_{1} I_{L} & -\left(\gamma+d_{A}+\mu_{A}\right)\end{array}\right)$

Evaluating Jacobian at Prosopis free equilibrium point we get: 
A Deterministic Mathematical Model for the Spread of Prosopis Juliflora Plants

$$
\left.J\right|_{P F E}(1)=\left(\begin{array}{ccccc}
-\mu_{L} & -\frac{\beta_{1} \Lambda_{1}}{\mu_{L}} & \gamma_{2} & 0 & 0 \\
0 & -\left(\mu_{L}+\omega_{L}\right) & 0 & 0 & 0 \\
0 & \omega_{L} & -\left(\gamma_{2}+\mu_{L}\right) & 0 & 0 \\
0 & -\frac{\beta_{2} \Lambda_{2}}{\mu_{A}} & 0 & -\mu_{A} & \gamma \\
0 & \frac{\beta_{1} \Lambda_{2}}{\mu_{A}} & 0 & 0 & -\left(\gamma+d_{A}+\mu_{A}\right)
\end{array}\right)
$$

eigenvalues of Jacobian are $\lambda_{1}=-\mu_{l}<0, \lambda_{2}=-\mu_{A}<0$;

Also in the second evaluation we get:

$$
\left.J\right|_{P F E}(2)=\left(\begin{array}{ccc}
\left(\mu_{L}+\omega_{L}\right) & 0 & 0 \\
\omega_{L} & -\left(\gamma_{2}+\mu_{L}\right) \mu_{L} & 0 \\
\frac{\beta_{1} \Lambda_{2}}{\mu_{A}} & 0 & \left(\gamma_{2}+d_{A}+\mu_{A}\right)
\end{array}\right)
$$

which implies $\lambda_{3}=-\left(\mu_{L}+\omega_{L}\right)<0, \lambda_{4}=-\left(\gamma_{2}+\mu_{L}\right)<0$ and $\lambda_{5}=-\left(\gamma+d_{A}+\mu_{A}\right)<0$

The Prosopis free equilibrium for each population is locally asymptotically stable if and only if the number of secondary infections, is less than unit, that is $R_{0}<1$ and this is fact because we got negative eigenvalues.

\subsection{Global stability of Prosopis free equilibrium point (PFE)}

The Lyapunov Method and LaSalle's Invariance Principle have been generally used to break down security of self-governing frameworks of differential conditions. Korobeinikov] [10,18] proposed unequivocal Lyapunov work which was utilized to break down SEIR and SEIS scourge model [19], developed a logarithmic Lyapunov capacity to examine Lotka-Volterra frameworks furthermore, later this capacity was applied by Korobeinikov [20] to dissect endemic equilibrium for SIR, SIRS and SIS pestilence models. Vargas-De-Leon [21] set forward the composite quadratic Lyaponuv capacity to dissect solidness of endemic equilibrium for SIR, SIRS and SIS scourge models and later developed a composite-Volterra capacity to break down endemic equilibrium for the model with backslide. In this work we adopt Lyapunov work. Under this part we study the global behavior of the Prosopis endemic equilibrium, $E^{*}$ for the model system (1).

Theorem 1. The endemic equilibrium point for the Prosopis model System (1) is asymptotically $\Omega$ if $R_{0}>1$ stable 


\section{Joel Simon, Silas Mirau and Livingstone S Luboobi}

Proof: We constructed an explicitly Lyapunov function for the model system (1) using $[10,18]$. Approaches as it is useful to the most of the Sophisticated Compartmental epidemiological models. In this approach, we construct Lyapunov function of the form:

$$
L=\sum a_{i}\left(X_{i}-X_{i}^{*} \ln X_{i}\right)
$$

where $a_{i}$ is a properly selected positive constant, $X_{i}$ is the population of the $i^{\text {th }}$ is the equilibrium level. We define the Lyapunov function candidate $V$ for the model System (1) as:

$$
L=\sum_{i=1}^{5} a_{i}\left(X_{i}-X_{i}^{*} \operatorname{In} X_{i}\right)
$$

$\mathrm{L}=a_{1}\left(S_{L}-S_{L}^{*} \ln S_{L}\right)+a_{2}\left(I_{L}-I_{L}^{*} \ln I_{L}\right)+a_{3}\left(R_{L}-R_{L}^{*} \ln R_{L}\right)+a_{4}\left(S_{A}-S_{A}^{*} \ln S_{A}\right)+$ $a_{5}\left(I_{A}-I_{A}^{*} \ln S_{A}\right)$ where $a_{1}, a_{2}, \ldots, a_{6}$ are positive constant. The time derivative of the Lyapunov function $\mathrm{L}$ is given by:

$$
\begin{aligned}
& \frac{\partial L}{\partial t}=a_{1} \frac{\partial V}{\partial S_{L}} \cdot \frac{\partial S_{L}}{\partial t}+a_{2} \frac{\partial V}{\partial I_{L}} \cdot \frac{\partial I_{L}}{\partial t}+a_{3} \frac{\partial V}{\partial R_{L}} \cdot \frac{\partial R_{L}}{\partial t}+a_{4} \frac{\partial V}{\partial S_{A}} \cdot \frac{\partial S_{A}}{\partial t}+a_{5} \frac{\partial V}{\partial I_{A}} \cdot \frac{\partial I_{A}}{\partial t} \\
& \frac{\partial L}{\partial t}=a_{1}\left(1-\frac{S_{L}^{*}}{S_{L}}\right)\left(\Lambda_{1}+\gamma_{2} R_{L}-\beta_{1} I_{A} S_{L}-\mu_{L} S_{L}\right)+a_{2}\left(1-\frac{I_{L}^{*}}{I_{L}}\right)\left(\beta_{1} I_{A} S_{L}-\mu_{L} I_{L}-\omega_{L} I_{L}\right)+ \\
& a_{3}\left(1-\frac{R_{L}^{*}}{R_{L}}\right)\left(\omega_{L} I_{L}-\gamma_{2} R_{L}-\mu_{L} R_{L}\right) \\
& +a_{4}\left(1-\frac{S_{A}^{*}}{S_{A}}\right)\left(\Lambda_{2}+\gamma I_{A}-\beta_{2} I_{L} S_{A}-\mu_{A} S_{A}\right)+a_{5}\left(1-\frac{I_{A}^{*}}{I_{A}}\right)\left(\beta_{2} I_{L} S_{A}-\gamma I_{A}-\left(d_{A}+\mu_{A}\right) I_{A}\right)
\end{aligned}
$$

At endemic equilibrium point:

$$
\begin{aligned}
\frac{\partial L}{\partial t}= & a_{1}\left(1-\frac{S_{L}^{*}}{S_{L}}\right)\left(\beta_{1} I_{A}^{*} S_{L}^{*}+\mu_{L} S_{L}^{*}-\beta_{1} I_{A} S_{L}+\mu_{L} S_{L}\right) \\
& +a_{2}\left(1-\frac{I_{L}^{*}}{I_{L}}\right)\left(\mu_{L} I_{L}^{*}+\omega_{L} I_{L}^{*}-\mu_{L} I_{L}-\omega_{L} I_{L}\right)+a_{3}\left(1-\frac{R_{L}^{*}}{R_{L}}\right)\left(\mu_{L} R_{L}^{*}-\mu_{L} R_{L}\right) \\
& +a_{4}\left(1-\frac{S_{A}^{*}}{S_{A}}\right)\left(\beta_{2} I_{L}^{*} S_{A}^{*}+\mu_{A} S_{A}^{*}-\beta_{2} I_{L} S_{A}+\mu_{A} S_{A}\right) \\
& +a_{5}\left(1-\frac{I_{A}^{*}}{I_{A}}\right)\left(\gamma I_{A}^{*}+\left(d_{A}+\mu_{A}\right) I_{A}^{*}-\gamma I_{A}-\left(d_{A}+\mu_{A}\right) I_{A}\right)
\end{aligned}
$$

Rearranging the terms, we get: 
A Deterministic Mathematical Model for the Spread of Prosopis Juliflora Plants

$$
\begin{aligned}
& \frac{\partial L}{\partial t}=a_{1}\left(1-\frac{S_{L}^{*}}{S_{L}}\right)\left[-\beta_{1} I_{A} S_{L}\left(1-\frac{I_{A}^{*} S_{L}^{*}}{I_{A} S_{L}}\right)-\mu_{L} S_{L}\left(1-\frac{S_{L}^{*}}{S_{L}}\right)\right] \\
& +a_{2}\left(1-\frac{I_{L}^{*}}{I_{L}}\right)\left[-\mu_{L} I_{L}\left(1-\frac{I_{L}^{*}}{I_{L}}\right)-\omega_{L} I_{L}\left(1-\frac{I_{L}^{*}}{I_{L}}\right)\right] \\
& +a_{3}\left(1-\frac{R_{L}^{*}}{R_{L}}\right)\left[-\mu_{L} R_{L}\left(1-\frac{R_{L}^{*}}{R_{L}}\right)\right]+a_{4}\left(1-\frac{S_{A}^{*}}{S_{A}}\right)\left[-\beta_{2} I_{L} S_{A}\left(1-\frac{I_{L}^{*} S_{A}^{*}}{I_{L} S_{A}}\right)-\mu_{A} S_{A}\left(1-\frac{S_{A}^{*}}{S_{A}}\right)\right] \\
& +a_{5}\left(1-\frac{I_{A}^{*}}{I_{A}}\right)\left[-\gamma I_{A}\left(1-\frac{I_{A}^{*}}{I_{A}}\right)-\left(d_{A}+\mu_{A}\right) I_{A}\left(1-\frac{I_{A}^{*}}{I_{A}}\right)\right]
\end{aligned}
$$

Through simplification of (13) we get:

$$
\begin{aligned}
& \frac{\partial L}{\partial t}=-a_{1} \mu_{l}\left(\frac{\left(S_{L}-S_{L}^{*}\right)^{2}}{S_{L}}\right)-a_{2}\left(\mu_{L}+\omega_{L}\right)\left(\frac{\left(I_{L}-I_{L}^{*}\right)^{2}}{I_{L}}\right) \\
& -a_{3} \mu_{L}\left(\frac{\left(R_{L}-R_{L}^{*}\right)^{2}}{R_{L}}\right)-a_{4} \mu_{A}\left(\frac{\left(S_{A}-S_{A}^{*}\right)^{2}}{S_{A}}\right)-a_{5}\left(\gamma+d_{A}+\mu_{A}\right)\left(\frac{\left(I_{A}-I_{A}^{*}\right)^{2}}{I_{A}}\right)+G(\Omega) \\
& \text { where } G(\Omega)=\frac{-a_{1} \beta_{1}\left(S_{L}-S_{L}^{*}\right)\left(I_{A} S_{L}-I_{A}^{*} S_{L}^{*}\right)}{S_{L}}-\frac{a_{4} \beta_{2}\left(S_{A}-S_{A}^{*}\right)\left(I_{L} S_{A}-I_{L}^{*} S_{A}^{*}\right)}{S_{A}}
\end{aligned}
$$

The function $G(\Omega)$ is a non-positive, thus $G(\Omega) \leq 0$ for all $\Omega$.Therefore $\frac{\partial L}{\partial t} \leq 0$ in $\Omega$ and is zero when $\Omega=\Omega^{*}$, since $\frac{\partial L}{\partial t} \leq 0$ in $\Omega$ is zero when $\Omega=\Omega^{*}$, which implies that the largest compact set in $\Omega$ when $\frac{\partial L}{\partial t}=0$ is singleton $\left\{\Omega^{*}\right\}$ which is the endemic equilibrium. By Lassalle's invariant principle $[10,18]$, it implies that the endemic equilibrium $\Omega^{*}$ is globally asymptotically stable in the interior of $\Omega$ when $R_{0}>1 . R_{0}$ depends on the interactions of infected land and susceptible animals and ingested animal and susceptible land. $R_{0}>1$ Occurs when susceptible animals interact with infected land and when ingested animals interacts with the susceptible animals. At this point, endemic equilibrium is stable when $R_{0}>1$. Therefore, these interactions with birth rate of animals makes the endemic equilibrium to be globally asymptotically stable.

\subsection{Sensitivity analysis}

Though the use of parameter values in Table 2 and the definition of equation (14) its sensitivity index is calculated as shown in Table 3 [24]. 
Joel Simon, Silas Mirau and Livingstone S Luboobi

Table 3: Parameters and their descriptions

\begin{tabular}{|c|c|c|c|}
\hline Parameter & Descriptions & value & Source \\
\hline$\Lambda_{1}$ & $\begin{array}{l}\text { Reclamation rate into the susceptible } \\
\text { acres of land portions population }\end{array}$ & 0.95 & [12] \\
\hline$\Lambda_{2}$ & $\begin{array}{l}\text { Per capita birth rate of animal } \\
\text { population }\end{array}$ & 0.902 & Estimated \\
\hline$\mu_{A}$ & $\begin{array}{l}\text { Per capita natural death rate of } \\
\text { animal population }\end{array}$ & 0.0167 & [22] \\
\hline$d_{A}$ & $\begin{array}{l}\text { Per capita death rate of animal } \\
\text { population induced by ingested seed }\end{array}$ & 0.005 & [11] \\
\hline$\mu_{L}$ & $\begin{array}{l}\text { Land portion used for constructions } \\
\text { project and occurrence of natural } \\
\text { calamities }\end{array}$ & 0.00548 & Estimated \\
\hline$\omega_{L}$ & $\begin{array}{l}\text { Rate of progression from invaded } \\
\text { land to the reclaimed land }\end{array}$ & 0.0035 & Estimated \\
\hline$\gamma$ & $\begin{array}{l}\text { Rate of recovery or restoration of } \\
\text { infested animal into susceptible } \\
\text { animal }\end{array}$ & 0.00005 & {$[12,14]$} \\
\hline$\gamma_{2}$ & $\begin{array}{l}\text { Rate of recovery or restoration of } \\
\text { invaded land population into } \\
\text { susceptible land }\end{array}$ & 0.070 & [23] \\
\hline
\end{tabular}

Under this section, we performed the forward sensitivity analysis of Reproduction number $R_{0}$ with respect to its parameters to determine which parameter is sensitive to the invasion of the plant on land. In finding a way of reducing the invasion of Prosopis juliflora plant, it is better to understand the proportional importance of factors that are reliable for the spread and eradication on the plant invasion. The forward sensitivity index of parameter $\mu$ with respect to basic Reproduction number $R_{0}$ is denoted by $\tau_{\mu_{i}}^{R_{0}}$.Using the approach in $[10,14,18]$.the normalized forward sensitivity index of a parameter $\mu_{i}$ with respect to Reproduction number $R_{0}$ is defined by:

$$
\alpha_{\mu_{i}}^{R_{0}}=\frac{\partial R_{0}}{\partial \mu_{i}} x \frac{\mu_{i}}{R_{0}}[24,25]
$$

Using the parameters in Table 2 and definition in equation (14), the sensitivity indices of reproduction number with respect to its parameters are given in Table 3.

Table 2: Sensitivity indices for $R_{0}$

\begin{tabular}{ccc}
\hline Parameter & Value & Sensitivity Index \\
\hline$\Lambda_{1}$ & 0.95 & +0.5000 \\
$\Lambda_{2}$ & 0.0902 & +0.5000 \\
$\beta_{1}$ & 0.000062 & +0.5000 \\
\hline
\end{tabular}


A Deterministic Mathematical Model for the Spread of Prosopis Juliflora Plants

\begin{tabular}{|c|c|c|}
\hline$\beta_{2}$ & 0.000059 & +0.5000 \\
\hline$\mu_{A}$ & 0.00237 & -0.6597 \\
\hline$\mu_{L}$ & 0.00548 & -0.8051 \\
\hline$\omega_{L}$ & 0.0035 & -0.1949 \\
\hline$d_{\mathrm{A}}$ & 0.00005 & -0.00034 \\
\hline Parameter & Value & Sensitivity Index \\
\hline$\gamma$ & 0.005 & -0.3369 \\
\hline
\end{tabular}

The positive indices show that the basic reproduction number is directly proportional to the values of its parameter. On the other hand, the negatives indices indicate that the basic Reproduction number is inversely proportional to the parameters.

In Table 3: $\Lambda_{1}, \Lambda_{2}, \beta_{1}, \beta_{2}$ have positive indices which means its production number increases as it increases and vice-versa. Under this situation, implies that the reproduction number is directly proportional to birth rate of animals, interaction between ingested animals and Susceptible land, in this case, it shows that as the interaction between ingested animal and susceptible land increases it leads to more spread of the Prosopis juliflora plants. However, the negative indexed parameters are: $\mu_{A}, \mu_{L}, \omega_{L}, d_{\mathrm{A}}$ and $\gamma$. Due to this result, the natural death rate of animals, land other uses rate most sensitive negative parameters of the model, this shows that the secondary infection of land by the plants will decrease as ingested animal decreases.

\section{Results and discussion}

This section explains more on the findings from the study after being implemented in the MATLAB ODE45 through Numerical simulation as it explained below.

\subsection{Numerical simulation}

In order to determine which parameter is sensitive to the spread of Prosopis juliflora plants done by sensitivity analysis, we simulate the model using the parameters found in Table 3

\section{i. The effects of Prosopis juliflora plants dynamics on land}

Figure 2 shows the simulation of Prosopis juliflora dynamics model which implanted in the MATLAB as the figure itself shows.

This is the dynamics of Prosopis juliflora plants which shows that the ingested animals increase from 150 to 240 for 8 months which leads to decreases of susceptible animals particularly from 150 to 135 animals for the mentioned period. Moreover, for the same period an infected land increased from 150 acres of land to 250 acres which resulted into decrease of susceptible land from 150 acres to 90 acres. This indicates that, there is high spread of Prosopis juliflora plants and therefore, control measures and strategies are needed to minimize or prevent the occurring situation. 
Joel Simon, Silas Mirau and Livingstone S Luboobi

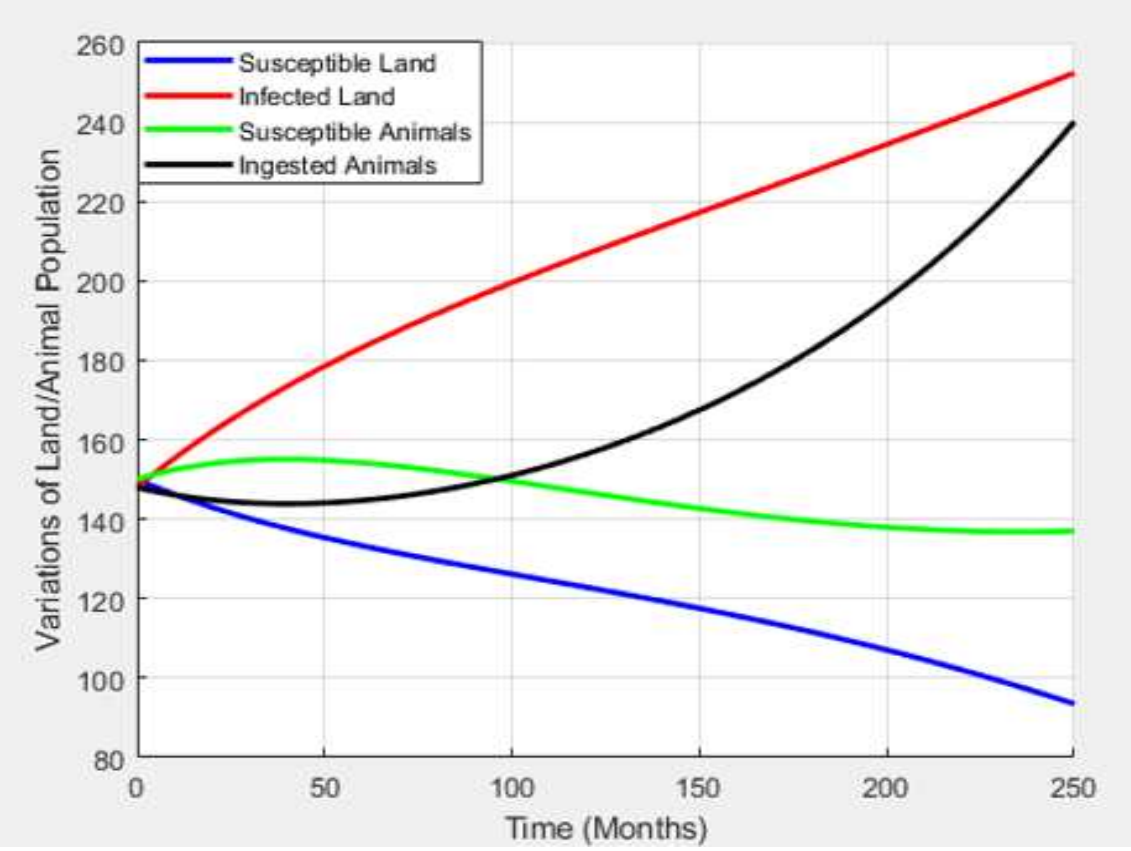

Figure 2: Dynamics of Prosopis juliflora plants on land and animal population

\section{ii. The effects of birth rate of animals in spreading the plant on Land}

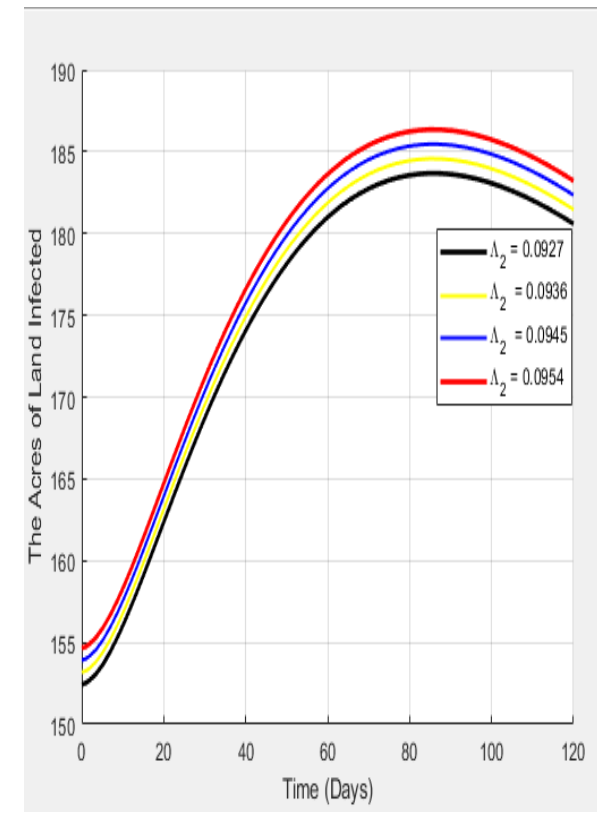

Figure 3: (a) Effects of variation of $\Lambda_{2}$ on land infected land

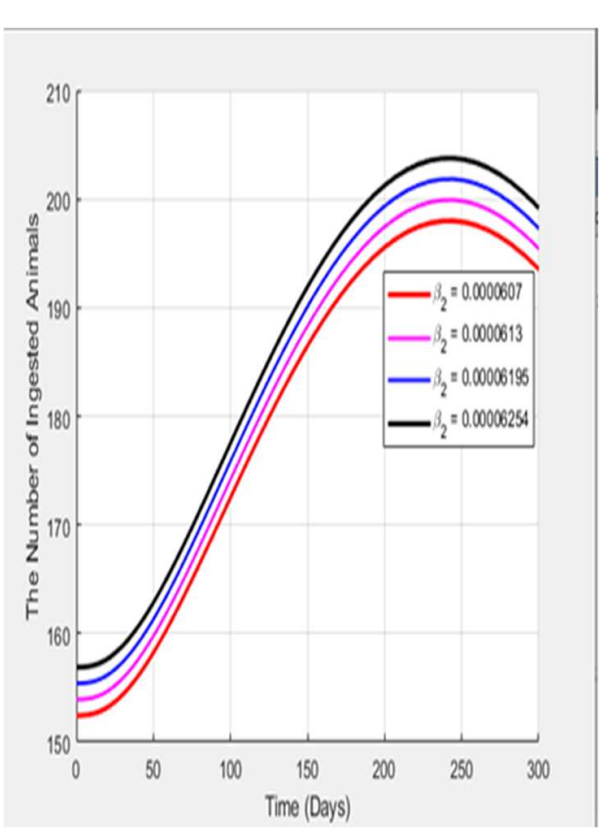

(b) Effects of variation $\beta_{2}$ with 
A Deterministic Mathematical Model for the Spread of Prosopis Juliflora Plants

The Figure 3 (a) shows that as per capita birth rate of animals increases it results into increase of infected land as from 152 acres of land to 187 acres within four months. Now this is very serious issue of an invasion of species and therefore there is need of extra efforts towards resolving the problem. On the other hand Figure 3 (b) shows that the interaction force between the susceptible animals and the infected land as it increases it results to an increase of ingested animals.

iii. The effects of ingested animals on susceptible animals and infected land on susceptible land
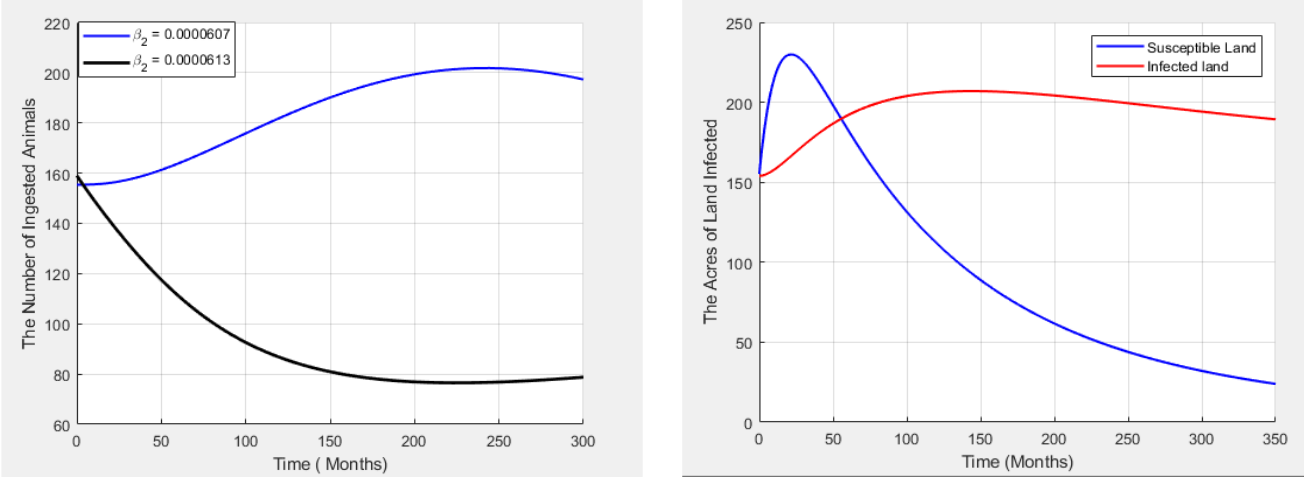

Figure 4: (a) Effects of Ingested Animals and Susceptible Animals (b) Effects of Infested Land and Susceptible

Figure 4(a) show that as animals ingested increases it leads to decreases of susceptible animals while Figure 4 (b) shows that as infected land increases it results to deceases of susceptible. In facts both Figures shows that its relationship are inversely propotional.

\section{iv. The effect of ingested Animals on Acres of land}

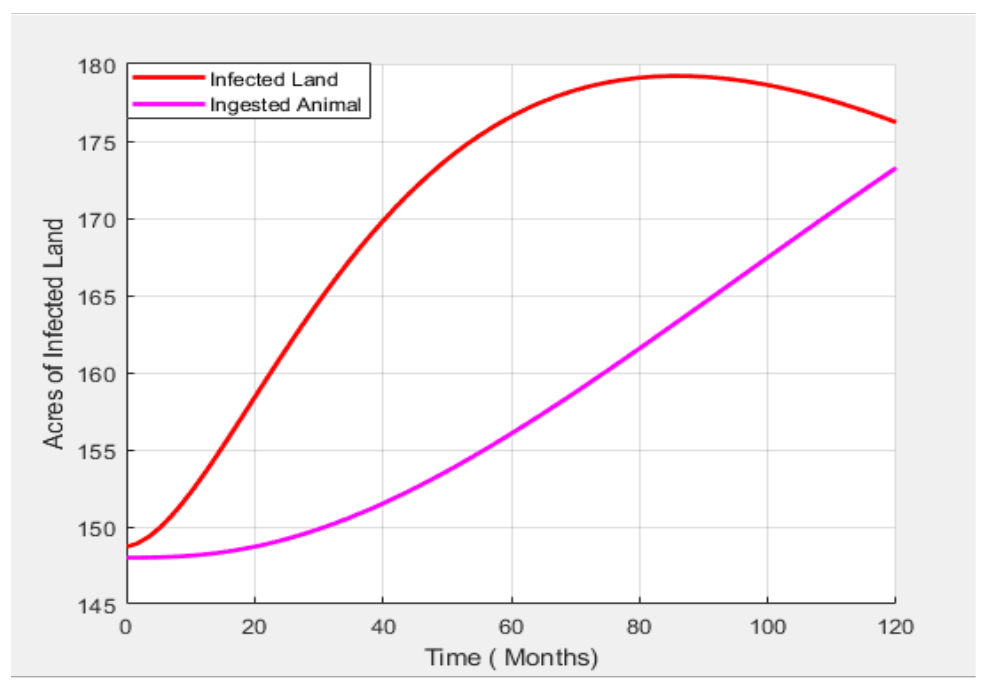

Figure 5: Variation of Ingested Animals on Acres of Land 


\section{Joel Simon, Silas Mirau and Livingstone S Luboobi}

Figure 5 show that as number of ingested animals increases it results to an increase of acres of infected land particularly from 148 acres to 178 acres which is equal to $20 \%$ rate of infection per month. In this case there is a need of extra efforts to rectify the situation on reducing or prevention on plants spread.

\section{v. Determination impact of each of parameters on the control of the spread of Prosopis juliflora plant}
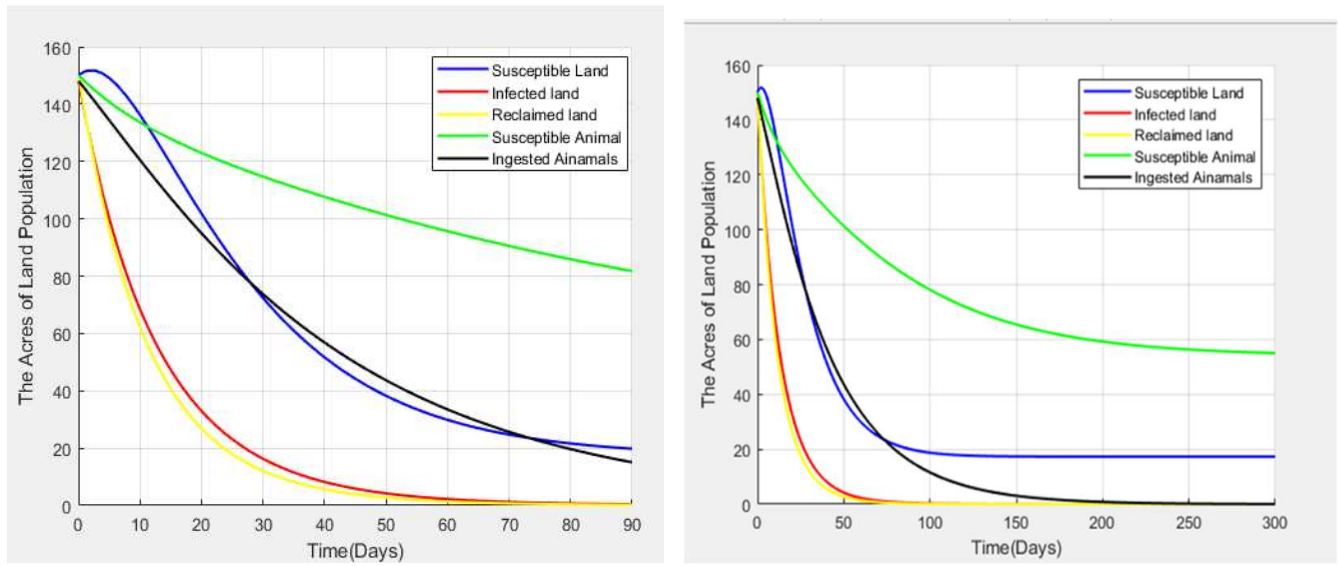

Figure 6: (a) Control of Prosopis juliflora within 1.5 months, (b) Control of Prosopis juliflora within 10 months

Figure 2 show that the spread was increasing before measures taken while Figure 6(a) showed that after applying measure of reducing and eradicating the plant within short time particular one month and half. Through applying the method for long time it managed to prevent a plant from spreading as shown in Figure 6(b) above.Morever this attained by varying the progression rate of invaded land to reclaimed land by $61.43 \%, \omega_{L}$ from 0.0035 to 0.035 through certain time as in this case ten months.

\section{Conclusion}

The study developed a deterministic model for the Prosopis juliflora dynamics which gives a better understanding on how plants invasion dynamics affect land and other native species. Moreover, basic reproduction number is computed and sensitivity analysis with their parameters performed as well. The study shows that when $R_{0}<1$ Prosopis free equilibrium is locally asymptotically stable. Furthermore the findings showed that the rate of plant spread due to interactions force on infested land and susceptible animals $\left(\beta_{2}\right)$ and to interactions force on infected animals and susceptible land $\left(\beta_{2}\right)$ with birth rate of animals on the reclaimed land are the most sensitive parameters on plants spread dynamics. As the interaction either between infested land and susceptible animals or susceptible land and infected animals are proportional to an increase rate of the plant spread. This is well supported by numerical simulation. The study recommends that strategies towards the control and prevention on plant invasion have to be implemented. 
A Deterministic Mathematical Model for the Spread of Prosopis Juliflora Plants

Acknowledgements. This work is supported by the United Republic of Tanzania through her Higher Education Student's Loans Board (HESLB) and Simons Foundations Fellowship in Botswana.

The authors are also thankful to the referees for their useful comments for improvement of the paper.

\section{REFERENCES}

1. C.Binggeli, Interior graphic standards, John Wiley \& Sons, 21 (2011).

2. A.Perkins, P.Benjamin, B. Mamsa and H.Alan, Evolution of dispersal and life history interact to drive accelerating spread of an invasive species, Ecology Letters, 16(8) (2013) 1079-1087.

3. S.Dzikiti, Schachtschneider, K.Naiken,V.Gush, G.Mosses and L.M.David, Water relations and the effects of clearing invasive Prosopis trees on groundwater in an arid environment in the Northern Cape South Africa, Journal of Arid Environments, 90 (2013) 103-113.

4. J.Leroux, M.Larrivee, L.Boucher, A.Hurford, J.Zuloaga, T.Kerr and F.Lutscher, Mechanistic models for the spatial spread of species under climate change, Ecological Applications 23(4) (2013) 815-828.

5. T.Shackleton, L.M.David, W.Van, W.Brian, Richardson and M.David, Towards a national strategy to optimise the management of a widespread invasive tree (Prosopis species; mesquite) in South Africa, Ecosystem Services, 27(2017) 242-252.

6. Haregeweyn, A.Tsunekawa, M.Tsubo, D.Meshesha and A. Melkie, Analysis of the invasion rate, impacts and control measures of Prosopis juliflora: a case study of Amibara District, Eastern Ethiopia, Environmental Monitoring and Assessment, 185(9) (2013) 7527-7542.

7. S.Mudavanhu, M.Blignaut, N.Vink, J.Crookes and N.Nkambule, An economic analysis of different land-use options to assist in the control of the invasive Prosopis (Mesquite) tree, African Journal of Agricuture and Resource Economics, 12(12) (2017) 366 - 411

8. L.Tilahun and A. Asfaw, Modeling the expansion of Prosopis juliflora and determining its optimum utilization rate to control its invasion in Afar Regional State of Ethiopia, International Journal of Applied Mathematical Research, 1(4) (2012) 726-743.

9. F.Obiri, Invasive plant species and their disaster-effects in dry tropical forests and rangelands of Kenya and Tanzania, Jàmbá: Journal of Disaster Risk Studies, 3(2) (2011) 417-428.

10. N.Nyerere, L.Luboobi, C.Mpeshe and M.Shirima, Mathematical model for the infectiology of brucellosis with some control strategies, New Trends in Mathematical Sciences, 4(7) (2019) 387-405.

11. O. Akinade, A. Afolabi, and E.Kimathi, Mathematical modeling and stability analyses of lassa fever disease with the introduction of the carrier compartment, Journal of Mathematical Theory and Modeling, 9(6) (2019) 45-62.

12. W.Aloyce and D.Kuznetsov, A mathematical model for the mlnd dynamics and sensitivity analysis in a maize Population, Asian Journal of Mathematics and Applications, 2017.

13. J.Kilawe, R. Mbwambo, C.Kajembe, E.Mwakalukwa, M.Amri, V.Mushi, M.Athumani, S.Eckert and R.Eschen, Mrashia: Prosopis invading pastures and 
Joel Simon, Silas Mirau and Livingstone S Luboobi

agricultural lands in Tanzania. The Woody Weeds Project INSIGHTS Report Mrashia: Prosopis has started invading pastures and agricultural lands in Tanzania, Woody invasive alien species in Eastern Africa Insights (2017).

14. I.Bimbiga, J.Irunde and D.Kuznetsov, Modeling malaria sensitive and resistant strains with superinfection, Commun. Math. Biol. Neurosci, 5(2019).

15. O.Diekmann, J.Heesterbeek and M.G.Roberts, The construction of next-generation matrices for compartmental epidemic models, Journal of the Royal Society Interface, 7(47) (2010) 873-885.

16. E.Lazarus, Lyapunov functions in epidemiological modeling, University of Namibia, (2018).

17. P.Van den Driessche and J.Watmough, Reproduction numbers and sub-threshold endemic equilibria for compartmental models of disease transmission, Mathematical biosciences, 180 (1-2) (2002) 29-48.

18. J.Irunde, L.S.Luboobi and Y.Nkansah-Gyekye, Modeling the effect of tobacco smoking on the in-host dynamics of HIV/AIDS, J. Math. Comput. Sci, 6(3) (2016) 406-436.

19. A.Ferrera, A.Pascual-García and U.Bastolla, Effective competition determines the global stability of model ecosystems, Theoretical Ecology, 10(2) (2017)195-205.

20. A.Khan, K.Iqbal, Y.Khan and E.Alzahran, A biological mathematical model of vectorhost disease with saturated treatment function and optimal control strategies, Mathematical Biosciences and Engineering, 17(4) (2020) 3972.

21. Q.Ding, Y.Liu, Y.Chen and Z.Guo, Dynamics of a reaction-diffusion SIRI model with relapse and free boundary, Mathematical Biosciences and Engineering, 17(2) (2019) 1659-1676.

22. J.Mapinda, G.G. Mwanga and V. Masanja, Modelling the transmission dynamics of banana xanthomonas wilt disease with contaminated soil, Journal of Mathematics and Informatics, 17 (2019) 113-129

23. Z.Tabo, L.S.Luboobi and J.Ssebuliba, Mathematical modelling of the in-host dynamics of malaria and the effects of treatment, Journal of Mathematics and Computer Science, 17(1) (2017) 1-21.

24. F.Kapange, J.I.Irunde and D. Kuznetsov, Modeling transmission dynamics of northerncorn leaf blight disease with seasonal weather variations, Journal of Mathematics and Informatics, (2019) 39.

25. Fikiri Lucas Matonya and Dmitry Kuznetsov, Modeling on the effects of drug abuse to the societies, Journal of Mathematics and Informatics, (2019). 